\title{
Panel 2 Report: Autonomic Communication Roadmap
}

\author{
Mikhail I. Smirnov \\ Fraunhofer FOKUS, \\ Kaiserin-Augusta-Allee 31, 10589 Berlin, Germany \\ Mikhail.Smirnov@fokus.fraunhofer.de
}

\begin{abstract}
Situated and Autonomic Communication (AC) research roadmap needs to be addressed from a mixture of viewpoints. What are the market drivers for AC? Can we really automate SLA? Does it help AC to radically depart from TCP/IP? Do we know all new requirements for networking software, auto- and re-configuration? How autonomics shall transform network management? What's the role of governance in autonomic control hierarchy, and do we know how hierarchy should emerge? These and similar questions were used to set the scene for the panel discussion on AC roadmap.
\end{abstract}

\section{Introduction}

Project $\mathrm{ACCA}^{1}$ coordinates the creation of a harmonised $\mathrm{R} \& \mathrm{D}$ programme to be implemented by the proactive initiative Situated and Autonomic Communication (S\&AC), a part of EU IST framework programme six and beyond. The goal of the programme is long-term foundational research in computer communications with the focus on studies in the area of network infrastructure self-organisation (self-management, self-healing, self-awareness, etc.). One of the major target applications is the design of a network element's autonomic behaviour exposed by innovative (cross-layer optimised, context-aware, and securely programmable) protocol stack in its interaction with numerous often-dynamic network communities. This is seen as the major vehicle for a new generation of ICT services to meet the requirements of information society.

The panellists were the following project participants: Mikhail Smirnov of Fraunhofer FOKUS in Germany (chair), Lidia Yamamoto of University of Basel in Switzerland, Spyros Denazis of University of Patras in Greece and Hitachi SAL in France, Simon Dobson of University College Dublin in Ireland, Ioannis Stavrakakis of NKUA in Greece, James Scott of Intel Corporation (UK) Ltd., David Lewis of Trinity College Dublin in Ireland, Jaouhar Ayadi of CSEM in Switzerland, and Serge Fdida of UPMC in France. The two invited speakers were Fabrizio Sestini of European Commission Future and Emerging Technologies,

The original version of this chapter was revised: The copyright line was incorrect. This has been corrected. The Erratum to this chapter is available at DOI: 10.1007/978-3-540-32993-0_29

${ }^{1}$ ACCA - Autonomic Communication Coordination Action, IST-6475 http://www. autonomic-communication.org/projects/acca/ 
and Nancy Alonistioti of University of Athens in Greece, also representing the IST integrated project E2R.

Prior to the panel the panellists have agreed that autonomic networks share a particular common characteristics. First, they are distributed and self-organized. Second, they must be elastic with regard to new services, goals and border conditions (Tschudin). Third, they must address the ongoing transfer of operational knowledge and decision-making authority from human operators to the system (Lewis).

The object of studies in S\&AC is the autonomic network element as it is affected by and affects other elements and the often numerous groups to which it belongs as well as network in general. A design methodology is needed that shall empower autonomic network elements with the abilities to understand how desired element's behaviours are learned, influenced or changed, and how, in turn, these affect other elements, groups and network.

\section{State of the Art in S\&AC}

After the initial white paper on AC [14] a number of conferences and journals have been considering S\&AC as a topic, on which submissions were solicited. The majority of publications though did not address the difference between IBM's autonomic computing and AC. The overview [13] has an excellent motivation for autonomic computing; it can be largely borrowed to motivate the $\mathrm{AC}$ research as well. However, certain care should be taken when borrowing the IBM's selfmanagement paradigm that is usually explained as "Monitor - Analyze - Plan Execute" sequence. Unpredictable traffic and network load behaviour in packet switched networks place autonomic decision making under the condition of deep uncertainty, where some governance needs to be provided either from a Knowledge Plane (KP) [16] in a form of behaviour rules or from dynamic network communities in a form of community context or fitness. In this case the "Analyze - Plan" could be seen as being outsourced to KP, while autonomic network element shall instantly act following the "Sense - Assess Risk - Behave" sequence, while concurrently at more relaxed time scale being also in communication with the KP and/or communities [1]. On a road to AC the research community has to build a new science of interaction (aka, science of interfaces [17]) addressing the above, and perhaps other approaches, finding the way to create, learn and influence behaviours, to detect and to assess risks, to understand how to apply policy- based management in these settings, etc. - all these to assure end-to-end services guarantees.

The state of the art in AC research is being shaped not only from the academic interest but also from projects funded by the industry and by the EU Commission. The Future and Emerging Technologies (FET) part of IST programme that has a record of proactive initiatives has started to prepare the S\&AC one in July 2003 with the four selected projects to start early 2006 [2]. FET views the S\&AC research as the answer to many networking challenges identified, such as increasingly high complexity of management, emergence of multi-technology paradigms 
(e.g. embracing ad hoc and sensor networks), pervasiveness and ubiquity of computing and communication in support of ambient intelligence, etc. with a strong emphasis on multi-disciplinarily research. The four S\&AC integrated projects that are set up to investigate the goal of task- and knowledge-driven, scalable, trustworthy, resilient, evolvable and society-friendly networking are BIONETS, ANA, Haggle, and CASCADAS.

The BIONETS (BIO-inspired NExt generation Services) project shall investigate a bio-inspired approach to localized communication services that should be able to evolve spontaneously, without centralised control. The project targets a communication system supporting millions of localized services in an environment consisting of billions of heterogeneous nodes, intermittently connected and extremely low-cost. The two types of nodes (static and mobile) are envisaged to form the project's peer-to-peer communication architecture, in which high-level services will adapt by evolution following the rules of genetics.

The ANA (Autonomic Network Architecture) project is addressing the architectural stress of the Internet. It will develop a novel network architecture that enables for flexible, dynamic and secure autonomic formation and adaptation of network elements and networks. Following the principles of atomisation, diffusion and sedimentation the project shall depart from the statically and globally layered protocol stacks aiming instead at dynamic flexible functional composition for wired and wireless networks. The project goal is to demonstrate the feasibility of situated and autonomic networking by 2010 .

The project Haggle wants to support transmission when end- to-end contemporaneous connectivity is not available, taking advantage of local and global connectivity; it will build on the model of search engines such as Google, but with no centralised services and no prerequisite of network connectivity; it has no ambition to become an alternative to global services.

The goal of the project CASCADAS (Component-ware for Autonomic Situation- aware Communications, and Dynamically Adaptable Services) is to define the underlying technology for a new generation of composite, highly distributed pervasive services that addresses the configuration and complexity problem at the level of resources and services. The project is therefore driven by the ambition of identifying a fundamental, uniform abstraction for situated and autonomic communication entities, at all levels of granularity, and across stack layers. This abstraction will be the cornerstone of CASCADAS's component model, in which four driving scientific principles will properly converge: situation awareness, semantic self-organisation, self-similarity, and autonomic componentware.

Not only FET funded projects but also mainstream IST projects are addressing very relevant technical goals. The integrated project E2R (End-to-End Reconfigurability) is a part of wireless world initiative; it considers reconfigurability as the enabler of seamless experience in all-IP infrastructure [3]. In a tough international competition the E2R is expanding the principles of software defined radio beyond cognitive radio and brings autonomous cognitive and proactive end-to-end reconfigurability to the telecom sector. The project sets itself to design a reconfiguration management plane based on an abstract view of a 
network or network element in the standard-friendly manner using the 3GPP integration reference point specification stages. Practically, the project has created a UML profile for reconfiguration that together with other results contributes to standards bodies like OMG and TMF.

Considering the above state of the art research plans and results as the starting point of S\&AC roadmap, the rest of this text will be structured in two parts - deliberations and S\&AC research challenges. Deliberations part first, addresses the risk and potential reasons of S\&AC failure [4], followed by reasoning originated within the eternal motivation for research - curiosity and knowledge enhancements [5]. Then, the myth of reduced complexity will be attacked [6]. The research challenges part examines selected facets of S\&AC and provides per facet a partial roadmap. This part starts with the governance dynamic [7], followed by scenario-based design [8], trends and promises in microelectronics area [9], stressing the need of applied S\&AC research [10], classical networking issues to be addressed in AC [11], and concludes with a phasing attempt of S\&AC roadmap.

\section{Deliberations}

Despite the visible success of $\mathrm{S} \& \mathrm{AC}$ as a research direction it is important to alert the community on the risk factors that eventually might downgrade the initiative to yet another hype; to have early understanding of the required depth of the research, and to unveil any myth that might break the research.

The three failure risk factors were identified in [4]: ignorance, fear, and selfstar [16]. The ignorance is bad because nobody knows what autonomics really is; at the same time, since nobody knows what autonomics is not, it might be good as well, for example more researchers will be attracted. The fear is bad because humans fear to loose control, and in fear of selfish behaviour of autonomic network will demand many control knobs, effectively preventing the idea of autonomics. From the positive viewpoint the fear will help to find fundamental limitations of the new technology. Self-star is risky since it might happen that its even very valuable solutions will open doors for new and serious problems that will require yet more effort to solve. The concern has been expressed that self-star could require even more human intervention than before thus perverting the idea of autonomics. Ironically, the "good news" about self-star is the risk that it becomes its own self- justification in an emergent way.

The panel presentation in [4] also proposed the cure for all the risks. To cure ignorance, the community needs to find a razor-sharp definition of AC; it's better to be too restrictive initially than to be too open or too late. To cure the fear, one needs to demonstrate that autonomic solutions are more robust than those relying on human intervention, which in turn requires research on novel ways of expressing "What we want?" instead of "How?". Finally, two things have been proposed as the cure for the risk of a self-justifying fate of self-star: a measure of autonomicity and the complexity handling. The former is to be inversely proportional to the amount of human intervention, the latter includes proper encapsulation. 
Correct treatment of complexity that requires early understanding of what is needed but is yet unknown and defines the necessary depth of research was addressed in [5]. Complexity in any system typically arises from two different sources: in intrinsic complexity of individual components, and the interaction complexity between components. In a software context these might be paraphrased as application complexity and system complexity respectively. While we have a reasonable understanding of applications, and their focus on addressing possibly complex but bounded and well-specified problem, we have significantly less understanding of the systems aspects especially in the presence of adaptation.

These interactions should be an object of study in their own right. We do not have a good understanding of component composition in software, as interactions between components are often surprising. We have even less understanding of the composition of adaptive components, whose adaptations may typically be expected to be antagonistic rather than synergistic with one component negatively impacting the adaptations of another. These aspects are typically addressed (in other domains) through control theory, but even here there is only a limited understanding of complex interactions that are sensitive to on-going conditions, and it is not clear that such models provide a good basis for software.

It is not enough for an adaptive system to adapt: it must exhibit the correct adaptation for the circumstances, retaining (and possibly optimising) some properties. This in turn implies an external semantic frame of reference within which issues such as optimality and trade-offs may be expressed and studied, in terms of the process which the system is involved in supporting. This goes beyond simple static descriptions of component interfaces.

A foundational science of composition will allow us to state and study both individual adaptations and their composition in a way that supports open adaptation while maintaining core properties.

In a myth unveiling fashion the [6] did negatively answer the question "Will autonomicity reduce management complexity?". The rational of autonomic computing adopted by S\&AC is to spread the cost of management to several entities; this does not imply that the overall complexity is reduced, From the history of consumer electronics we learn that management complexity can be reduced by integration. On contrary, S\&AC attempts to disintegrate a system, it increases the number of interfaces we need to manage, it adds anarchy-inducing autonomicity. This may lead to spreading the cost between multiple entities however the overall management complexity increases. Contrary to automation that reduces management complexity, increases performance, capacity and efficiency, the autonomicity, as a disintegration mechanism increases the complexity. From a positive side [6] observes that autonomicity is not an invention of the S\&AC community, it is emerging as the network naturally disintegrates following the process of shifting of the ownership of resources to autonomic entities (contributors). From this viewpoint the AC research has the two major challenges. First, behaviour management since the behaviour is the single most defining characteristic of an autonomous element that follows its own laws or lack of those. Second, interaction management (aka, science of interfaces [17]) that can be grounded 
by borrowings from the ecology by the notions of individualism (organisms), behaviours (rational, irrational, changing, random, unpredictable, etc.), interactions, equilibrium (slow changes), and evolution. In this new science complexity will be managed without central authorities and global rules.

\section{Research Challenges}

Stating that autonomic system must be governed rather than managed [7] argues that this will require two things. First, operational goals and constraints have to be expressed as policies, and second the process of definition of these policies by humans will need on-going human understanding of adaptive space and its governance potential. The latter can be formalised as a governance space - an operationally accessible portion of an adaptive space, while adaptive space in turn can be represented by contextual space with possible adaptive behaviours. There are certain restrictions within governance space, for example grouping must reflect organisational and social policy-making, and take into account such non-functional aspects of the latter as stability, responsiveness and potential for conflict.

Governance is a dynamic two-way process within a system hierarchy: delegation of decision making authority propagates governance policies downstream; escalation of decision making due to governance space violations propagates detected policy conflicts upstream. Note that violations of adaptive space lead to a semantic mismatch. The research vision here is to find ways of applying the governance process recursively between communities of agents within a system. This vision translates into the following set of research objectives. First, since the governance process needs to be managed, one needs to build a (e.g. community based) policy management mechanism with a requirement for fast stabilisation of policy set for a given governance space. Second, since the governance space is volatile due to changing contexts, service offerings, and value chains a handling mechanism for a governance space is needed supported by semantic mappings to convey adaptive space.

Roadmap-wise [7] suggests as the first step to gain understanding of human decision-making dynamics in a small cross-disciplinary research project. This can be followed by a larger project to establish benchmarks for assessing governance effectiveness and explaining benefits. As the third step a number of projects could develop forms of adaptive and governance spaces, build and evaluate solutions for the governance dynamic in different domains, such as communications, pervasive computing, electronic markets, and collaborative spaces.

From a traditional management perspective [8] argues that autonomicity is a property of evolution in the making that revolves around an intelligent selfcentric control loop "Collect - Decide - Enforce" and should be addressed in conjunction with application field. In network management Collect translates into monitoring and building a network picture, helps to achieve self-awareness; Decide translates into inference, i.e. problem diagnosis process and into planning - a process of selecting a solution; Enforce translates into deployment i.e. adding functionality and configuration, i.e. changing the behaviours. 
Autonomicity without tangible and universal scenarios is meaningless; the above control loop is lifeless without semantic languages that enable exchange between loop entities that operate at different levels and in different contexts. Collectively, these semantic languages describe purposeful behaviours of network and services composed of components. For example, with emerging modular router architectures where control and forwarding are separated the support for components is provided.

Roadmap-wise [8] suggests in the first phase to select a characteristic application field with a set of representative scenarios, and to select existing functionality to be transformed into an autonomic one. This can be done by defining abstractions for the intelligent control loop tailored for this functionality and suitable for low-level programmability, assuming modular router as a target platform. This can be followed by large-scale trials and testbeds.

Modular architectures are enablers for autonomicity, however they are in turn enabled by the progress in microelectronics, manufacturing and power supply. The [9] provides an outlook into 10-15 years from now with the goal to outline the wireless and mobile communication landscape in that future. Humans are in the centre of this future of communication, surrounded by ambient intelligence and autonomic networking. It is envisaged that by 2020 microelectronics industry will reach the level of 1 million MIPS $^{2}$ per 10 Watt, which is expected to be sufficient for autonomic communication demands. The basic element - an autonomic component - will be a universal building block for autonomic applications and systems. It will be a modular unit with specified interfaces, clear context dependencies and self-management mechanisms that shall manage behaviour within actual constraints based on policies and rules. Policy will govern not only self-management of individual components but also interactions between components, including agreements establishment towards consistency, robustness and system self-management.

Contributing to the interface science discussion [9] argues that today's physical interfaces to network and equipment might disappear at the time of autonomic communication being replaced by ubiquitous short-range radio communication. In this environment precise localisation, sensing, sounding, etc. will foster the ability to adapt to changes in traffic load, service, even functionality required. With ultra-low power consumption self-sustained operation over years is predictable, combined with ultra small size and low cost it brings autonomic communication to the size we can swallow.

However we should not need to wait until 2020 to start working on situated and autonomic communication; [10] calls for applied S\&AC to solve real-life problems, which does not preclude long-term research. Even more, perhaps theory and practice must work more closely to constantly verify theoretic results in experiments. Without practically building new technology we can't understand the constraints; building actually means transfer of results between groups of developers. Technology evaluation requires more sets of measurement data available publicly; this will facilitate development of realistic models and trace-based

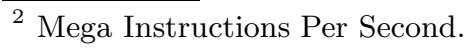


evaluation largely in place of simulation. Since applied S\&AC calls for higher involvement of community members it is necessary to create proper incentives including funding and programme committees.

As observed by [11] foundation of the Internet technology can be compared with the foundation of S\&AC; this view perhaps is helpful in the understanding of $\mathrm{S} \& \mathrm{AC}$ roadmap. There are always similar forces (applications, regulation, economy, management) that are shaping the R\&D learning curve: early solutions tend to have higher complexity, while solutions in use converge over time to the level of complexity that is conformant to the level of understanding of the problem area, though only until the next innovation cycle starts. The state of the art understanding in $\mathrm{AC}$ is characterised in [11] as the one that lacks a single solution though has a clear networking focus proposing a very ambitious future for communication. While focussing mainly on self-star properties the AC will impact many networking issues: naming and addressing for autonomic entities, data gathering and knowledge management including interactions with the environment, soft-layering leading to time-dependant architectures, interoperability in multiple contexts, composition and behaviour modelling, service management and adaptation.

\section{Conclusions}

Any roadmap is a tool for strategy development, S\&AC roadmap needs to show a path to full $\mathrm{AC}$, and perhaps even beyond starting with the tailored description of "now". As it is outlined in [12] the current situation is characterised as proliferation of brittle systems that often require babysitting; the Internet appears to end systems as a black box, its end systems are vulnerable to blue screens and viruses. The full S\&AC promises fully autonomic operation at many levels, so that underlying systems conspire to do what we want automatically, perhaps based on self-star. We do not know yet how to characterise the life beyond full S\&AC, the [12] mentions intelligence, autonomy and creation and suggests four stages of the roadmap.

The zero phase is the one that is happening now, its main goal is to agree on a definition, to continue the dissemination activities and to work closely with funding bodies on improving the awareness. This will soon be followed by the first phase, in which the four funded projects mentioned above should investigate their domains. Interleaved with this, the second phase should embrace the most mature S\&AC topics as part of the mainstream IST research, in EU as framework programme seven (2007-2013), and worldwide as activities coordinated by ACF, IEEE, etc; while long-term outstanding S\&AC topics would still remain in FET. The third phase should continue the S\&AC research but should also expand to the society to start using mature solutions.

The strongest consensus during the zero phase is the S\&AC definition need; we must agree on what is S\&AC and what is not. Tentatively, [12] suggest to agree that S\&AC systems are those that do what we want without direct human supervision using for this context information and knowledge, policies 
and conflict resolution, embedded performance evaluation and feedback; these systems do things by themselves, meaning that their components self-organise and use emergence of e.g. control hierarchies as a part of self-organisation. The S\&AC systems are self-managed; this property is seen as an integral characteristic comprised of abilities to be self-protected, including security and immunity, self-diagnosed including detection of failures and conflicts, self-healed, including correction, repair and recover actions, self-configured including updates of functionality at several levels, self-optimised including self-adaptation and evolvability, and self-deployed including self-deprecation.

What is not S\&AC then? Perhaps we should agree on a fuzzy metric of autonomicity that will be computed as a weighted sum of different criteria in the definition and will be inversely proportional to the amount of human intervention. However the risk is to have too wide scope for S\&AC and to be hardly distinguishable from pervasive and ubiquitous computing, from autonomic computing, and from networking. The phase one projects might provide answers in the following ascending sequence along the imaginable axis comprised of $\boldsymbol{r i s k}$, time and futuristic orientations. The clear start is to be found within wireless and broadly defined opportunistic networking (project Haggle); these and other advances are likely to be abstracted to the S\&AC networking architecture by project ANA; the next mark on the imaginable axis is to be provided by project CASCADAS that shall address pervasive S\&AC, bring it based on its component model in line with context and knowledge. Finally, the project BIONETS is targeting evolutionary protocols and services reflecting socio-economic models. In general, the first phase outcomes are expected to be both well established theoretical foundations for S\&AC and practical case studies covering handheld solutions, meetings and conferences support, and home platforms.

The society in general is expected to use, to enjoy and to benefit from S\&AC by radical increase of productivity levels in engineering (adaptation and evolution), software synthesis (eternal software, autocatalytic systems), and even in research itself (e.g. in complex systems and emergence). However for this to happen S\&AC has to receive much more attention from funding bodies, namely to become a part of mainstream ICT. Immediate ICT benefits will be technology- and society-wise. Technology-wise examples are improved naming and addressing as opposed to current DNS and DHCP, and new communication services which are not limited by the end/to-end obligation. Society-wise, higher usability levels of ICT solutions, higher acceptance rates and better addressed socio-economic issues.

\section{References}

1. Mikhail Smirnov, Autonomic Communication Roadmap, WAC 2005 Panel overview, http://cgi.di.uoa.gr/ istavrak/PDF_presentations_WAC/Panel2_ WAC2005_Smirnov.pdf

2. Fabrizio Sestini, Situated and Autonomic Communications in FET, WAC 2005 Panel presentation, http://cgi.di.uoa.gr/ istavrak/PDF_presentations_WAC/ Panel2_Sestini_WAC2005.pdf 
3. Nancy Alonistioti, Management of Reconfigurability, WAC 2005 Panel presentation http://cgi.di.uoa.gr/ istavrak/PDF_presentations_WAC/Panel2_Alonistioti_ WAC2005.pdf

4. Christian Tschudin, Lidia Yamamoto, Reasons why AC may fail, and how to prevent it, WAC 2005 Panel position statement, http://cgi.di.uoa.gr/ istavrak/ PDF_presentations_WAC/Panel2_ubasel_WAC2005_red.pdf

5. Simon Dobson, Everything interesting is composition, WAC 2005 Panel position statement, http://cgi.di.uoa.gr/ istavrak/PDF_presentations_WAC/PAnel2_ WAC2005_Dobson.pdf

6. Ioannis Stavrakakis, Will Autonomicity reduce management complexity? No! WAC 2005 Panel position statement, http://cgi.di.uoa.gr/ istavrak/PDF_ presentations_WAC/Panel2_WAC2005_Stavrakakis.pdf

7. David Lewis, An Autonomic Governance Dynamic, WAC 2005 Panel position statement, http://cgi.di.uoa.gr/ istavrak/PDF_presentations_WAC/Panel2_ wac05-Lewis.pdf

8. Spyros Denazis, Autonomicity is a property, WAC 2005 Panel position statement, http:// cgi.di.uoa.gr/ istavrak/PDF_presentations_WAC/Panel2_ WAC2005_Hitachi.pdf

9. John Farserothu, Jaouhar Ayadi, Communication 2020 and beyond - on the road to Autonomic Communication, WAC 2005 Panel position statement, http:// cgi.di.uoa.gr/ istavrak/PDF_presentations_WAC/Panel2_WAC2005_CSEM.pdf

10. James Scott, Applied Situated and Autonomic Communications, WAC 2005 Panel position statement, http://cgi.di.uoa.gr/ istavrak/PDF_presentations_ WAC/Panel2_jscott- wac2005.pdf

11. Serge Fdida, ACCA, A Networking View, WAC 2005 Panel position statement, http://cgi.di.uoa.gr/ istavrak/PDF_presentations_WAC/Panel2_WAC2005 _Fdida.pdf

12. Lidia Yamamoto, Christian Tschudin, Autonomic Communication Roadmap Proposal, WAC 2005 Panel position statement, http://cgi.di.uoa.gr/ istavrak/PDF_ presentations_WAC/Panel2_ubasel_WAC2005_green.pdf

13. Autonomic Computing Overview, IBM, on line at http://www.research.ibm.com/ autonomic/overview/elements.html

14. Autonomic Communication: Research Agenda for a New Communication Paradigm, white paper, Fraunhofer FOKUS 2003, on-line at http://www. autonomic-communication.org/publications/doc/WP_v02.pdf

15. David D. Clark, Craig Partridge, J. Christopher Ramming, and John T. Wroclawski, A Knowledge Plane for the Internet, In Proceedings of SIGCOMM '03, 2003.

16. Ozalp Babaoglu, Ma'rk Jelasity, Alberto Montresor, Christof Fetzer, Stefano Leonardi, Aad van Moorsel, and Maarten van Steen, editors., Self-Star Properties in Complex Information Systems, volume 3460 of Lecture Notes in Computer Science, Hot Topics. Springer-Verlag, May 2005.

17. Paul Spirakis, Complexity vs Autonomicity, WAC 2005 Panel position statement,http:// cgi.di.uoa.gr/ istavrak/PDF_presentations_WAC/Panel1_wac_ Spirakis.pdf 